\title{
Cohomogeneity one hypersurfaces of Euclidean Spaces
}

\author{
Francesco Mercuri, Fabio Podestà, José A. P. Seixas and Ruy Tojeiro
}

\begin{abstract}
We study isometric immersions $f: M^{n} \longrightarrow \mathbb{R}^{n+1}$ into Euclidean space of dimension $n+1$ of a complete Riemannian manifold of dimension $n$ on which a compact connected group of intrinsic isometries acts with principal orbits of codimension one. We give a complete classification if either $n \geq 3$ and $M^{n}$ is compact or if $n \geq 5$ and the connected components of the flat part of $M^{n}$ are bounded. We also provide several sufficient conditions for $f$ to be a hypersurface of revolution.
\end{abstract}

Mathematics Subject Classification (2000). 53A07, 53C42.

Keywords. Cohomogeneity one manifolds, hypersurfaces.

\section{Introduction}

An interesting problem in submanifold theory is to study isometric immersions $f: M^{n} \longrightarrow \mathbb{R}^{N}$ into Euclidean space of a connected complete Riemannian manifold of dimension $n$ acted on by a closed connected subgroup of its isometry group Iso $\left(M^{n}\right)$. This study was initiated by Kobayashi [8], who proved that if $N=n+1$ and $M^{n}$ is compact and homogeneous, i.e., Iso $\left(M^{n}\right)$ acts transitively on $M^{n}$, then $f\left(M^{n}\right)$ must be a round sphere.

In this paper we consider isometric immersions $f: M^{n} \longrightarrow \mathbb{R}^{n+1}$ of a complete Riemannian manifold $M^{n}$ on which a compact, connected subgroup $G$ of $\operatorname{Iso}\left(M^{n}\right)$ acts with maximal dimensional orbits of codimension one. We call $f$ a hypersurface of $G$-cohomogeneity one. Observe that the group $G$ may not be realizable as a group of extrinsic isometries of the ambient space. For instance, consider the cohomogeneity one action of $\mathrm{SO}(n)$ on $\mathbb{R}^{n}$ and isometrically immerse $\mathbb{R}^{n}$ into $\mathbb{R}^{n+1}$ as a cylinder over a plane curve. However, such examples can only arise if $f$ is not rigid. Recall that $f$ is rigid if any other isometric immersion $\tilde{f}: M^{n} \rightarrow \mathbb{R}^{n+1}$ differs from $f$ by an isometry of $\mathbb{R}^{n+1}$.

Examples of cohomogeneity one hypersurfaces may be obtained as follows. Start with a cohomogeneity two compact subgroup $G \subset \mathrm{SO}(n+1)$, so that the orbit space $\mathbb{R}^{n+1} / G$ is a two dimensional manifold, possibly with boundary. Now consider 
a curve that is either contained in the interior of $\mathbb{R}^{n+1} / G$ or meets its boundary orthogonally. Then the inverse image of such a curve by the canonical projection onto the orbit space is a cohomogeneity one hypersurface. We shall call these examples the standard examples. Among them, the simplest ones are the hypersurfaces of revolution, which are invariant by the action of $\mathrm{SO}_{l}(n+1)$, the subgroup of $\mathrm{SO}(n+1)$ that fixes a straight line $l$.

Our main result states that, under natural global assumptions, the standard examples comprise all cohomogeneity one hypersurfaces.

Theorem 1.1. Let $f: M^{n} \longrightarrow \mathbb{R}^{n+1}$ be a complete hypersurface of $G$-cohomogeneity one. Assume either that $n \geq 3$ and $M^{n}$ is compact or that $n \geq 5$ and the connected components of the flat part of $M^{n}$ are bounded. Then $f$ is either rigid or a hypersurface of revolution. In particular, $f$ is a standard example.

We also provide several sufficient conditions for a hypersurface of $G$-cohomogeneity one as in Theorem 1.1 to be a hypersurface of revolution.

Theorem 1.2. Under the assumptions of Theorem 1.1, any of the following conditions implies that $f$ is a hypersurface of revolution:

(i) there exists a principal orbit with positive curvature;

(ii) there exists a principal orbit that is totally geodesic in $M^{n}$;

(iii) the principal orbits are umbilical in $M^{n}$;

(iv) $n \neq 4$ and there exists a principal orbit that is homeomorphic to a sphere.

Moreover, in this case $G$ is isomorphic to one of the closed subgroups of $\mathrm{SO}(n)$ that act transitively on $S^{n-1}$.

Theorem 1.2 generalizes and gives new (and shorter) proofs of various known results. Namely, it was proved under condition (iii) in [12] in the compact case for $n \geq 4$ and later in [9] in the general case (even for $n=3,4$ ). It was also proved in [4] (resp., [2]) in the compact case for $n \geq 5$ (resp., $n \geq 4$ ) under the assumption that all orbits have positive (resp., constant) sectional curvature. We also point out that closed subgroups of $\mathrm{SO}(n)$ that act transitively on the sphere are completely classified (cf. [7], p. 392).

\section{The proofs}

Given an isometric immersion $f: M^{n} \longrightarrow \mathbb{R}^{n+1}$, let $A_{\xi_{p}}$ denote the shape operator of $f$ at $p \in M^{n}$ with respect to a normal vector $\xi_{p} \in T_{p}^{\perp} M^{n}$, that is, the symmetric endomorphism of $T_{p} M^{n}$ given by $A_{\xi_{p}} X=-\tilde{\nabla}_{X} \xi$ for any $X \in T_{p} M^{n}$, where $\xi$ is 
a smooth local normal vector field extending $\xi_{p}$ and $\tilde{\nabla}$ stands for the derivative of $\mathbb{R}^{n+1}$. Recall that the relative nullity subspace of $f$ at $p \in M^{n}$ is the kernel of $A_{\xi_{p}}$. It is well-known that on any open subset of $M^{n}$ where the relative nullity subspaces of $f$ have constant positive dimension, they define a smooth distribution whose leaves (called the leaves of relative nullity) are mapped by $f$ onto open subsets of affine subspaces of $\mathbb{R}^{n+1}$.

Our approach to the study of hypersurfaces of cohomogeneity one is based on the following variant due to Ferus of a rigidity theorem of Sacksteder [14].

Theorem 2.1. Let $f, \tilde{f}: M^{n} \longrightarrow \mathbb{R}^{n+1}$ be isometric immersions of a complete Riemannian manifold of dimension $n \geq 3$. If there exists no complete leaf of relative nullity of dimension $n-1$ or $n-2$ (in particular if $M^{n}$ is compact), then the shape operators of $f$ and $\tilde{f}$ satisfy $A(p)= \pm \widetilde{A}(p)$ for every $p \in M^{n}$. As a consequence, if the subset of totally geodesic points of $f$ does not disconnect $M^{n}$ then $f$ is rigid.

The relation between the shape operators of $f$ and $\tilde{f}$ in the statement means, more precisely, that $\tilde{A}_{\psi\left(\xi_{p}\right)}= \pm A_{\xi_{p}}$ for any $p \in M^{n}$ and for any $\xi_{p} \in T_{p}^{\perp} M_{f}^{n}$, where $\psi: T^{\perp} M_{f}^{n} \rightarrow T^{\perp} M_{\tilde{f}}^{n}$ is one of the two vector bundle isometries between the normal bundles of $f$ and $\tilde{f}$.

By means of Theorem 2.1 we now derive the following result for hypersurfaces of $G$-cohomogeneity one, which is the main tool for the proofs of Theorems 1.1 and 1.2. We refer to [1] and the references therein for the basic facts on cohomogeneity one manifolds that are used in the sequel.

Proposition 2.2. Let $f: M^{n} \longrightarrow \mathbb{R}^{n+1}$ be a complete hypersurface of $G$-cohomogeneity one. If either $f$ is rigid or there exists no complete leaf of relative nullity of $f$ of dimension $n-1$ or $n-2$ (in particular if $M^{n}$ is compact), then

(i) $B$, the set of totally geodesic points of $f$, is $G$-invariant.

(ii) There exists a Lie group homomorphism $\Psi: G \longrightarrow \mathrm{SO}(n+1)$ such that $f \circ g=$ $\Psi(g) \circ f$ for every $g \in G$, that is, $f$ is $G$-equivariant.

(iii) If $\Sigma$ is a principal orbit of $G$, then $f(\Sigma)$ is a principal orbit of the action of $\widetilde{G}=\Psi(G)$ on $\mathbb{R}^{n+1}$. In particular, $f(\Sigma)$ is an isoparametric hypersurface of a sphere.

(iv) If $f(\Sigma)$ is a round sphere for some principal orbit $\Sigma$ of $G$, then $f$ is a hypersurface of revolution and $\Psi$ is a monomorphism. In particular, $G$ is isomorphic to one of the closed subgroups of $\mathrm{SO}(n)$ that act transitively on $S^{n-1}$.

Proof. Given $g \in G$, let $A^{g}$ denote the shape operator of $f \circ g$. If $f$ is rigid then $A^{g}=A$ for every $g \in G$. We claim that this is also the case if there exists no complete 
leaf of relative nullity of $f$ of dimension $n-1$ or $n-2$. In fact, on one hand we have

$$
g_{*}(p) \circ A^{g}(p)=A(g(p)) \circ g_{*}(p) \text { for each } p \in M .
$$

This implies that for each fixed $p \in M^{n}$ the map $\phi_{p}: G \rightarrow \operatorname{End}\left(T_{p} M^{n}\right)$ given by

$$
\phi_{p}(g)=A^{g}(p)=\left(g_{*}(p)\right)^{-1} \circ A(g(p)) \circ g_{*}(p)
$$

is continuous. On the other hand, it follows from Theorem 2.1 that for each $p \in M^{n}$ either $A^{g}(p)=A(p)$ or $A^{g}(p)=-A(p)$. We obtain that $\phi_{p}$ is a continuous map taking values in $\{A(p),-A(p)\}$. Since $G$ is connected and $\phi_{p}(\mathrm{I})=A(p)$, our claim follows.

In particular, the set $B^{g}$ of totally geodesic points of $f \circ g$ coincides with $B$ for every $g \in G$. In view of (1), this is equivalent to saying that $B$ is $G$-invariant. Moreover, by the Fundamental Theorem of Hypersurfaces, for each $g \in G$ there exists $\tilde{g} \in \operatorname{Iso}\left(\mathbb{R}^{n+1}\right)$ such that $f \circ g=\tilde{g} \circ f$. It now follows from standard arguments (cf. [12]) that $\Psi: G \longrightarrow$ Iso $\mathbb{R}^{n+1}, \Psi(g)=\tilde{g}$, is a Lie-group homomorphism whose image lies in (a conjugacy class of) $\mathrm{SO}(n+1)$, because it is compact (and hence has a fixed point) and connected. Assertion (iii) now follows from (ii).

Finally, if $f(\Sigma)$ is a round sphere for some principal orbit $\Sigma$ of $G$ then, since $G$ is connected, it must fix the line $\ell$ orthogonal to the linear span of $f(\Sigma)$. Hence $f$ is a hypersurface of revolution with $\ell$ as axis. Moreover, the restriction of $f$ to $\Sigma$ must be injective. Since $f \circ g=\Psi(g) \circ f$ for any $g \in G$, if $\Psi(g)=I \in \operatorname{SO}(n+1)$ for some $g \in G$ we obtain that $g(y)=y$ for all $y \in \Sigma$. Now, since $\Sigma$ is a principal orbit, this implies that, for every $y \in \Sigma, g_{*}$ acts trivially on the normal space at $y$ to the inclusion of $\Sigma$ into $M^{n}$. As a consequence, if $\gamma: \mathbb{R} \rightarrow M^{n}$ is a normal geodesic through $y \in \Sigma$, i.e., a complete geodesic that crosses $\Sigma$ (and hence any other $G$-orbit) orthogonally, then $g$ fixes any point of $\gamma(\mathbb{R})$. Since every point of $M^{n}$ lies in a normal geodesic through a point of $\Sigma$, we obtain that $g=I \in G$, and the last assertion in (iv) follows.

Our next result classifies complete hypersurfaces of $G$-cohomogeneity one with dimension $n \geq 5$ that carry a complete leaf of relative nullity of dimension $n-2$.

Proposition 2.3. Let $f: M^{n} \longrightarrow \mathbb{R}^{n+1}, n \geq 5$, be a complete hypersurface of $G$ cohomogeneity one. If there exists a complete leaf of relative nullity of dimension $n-2$ then $M^{n}=S^{2} \times \mathbb{R}^{n-2}$ and $f$ splits as $f=i \times \mathrm{id}$, where $i: S^{2} \rightarrow \mathbb{R}^{3}$ is an umbilical inclusion and id: $\mathbb{R}^{n-2} \rightarrow \mathbb{R}^{n-2}$ is the identity map. In particular, $f$ is rigid.

Proof. Since $M^{n}$ carries a complete leaf of relative nullity $\mathcal{F}$, it can not be compact. Thus the orbit space $\Omega=M^{n} / G$ is homeomorphic to either $\mathbb{R}$ or $[0, \infty)$. Moreover, if $\pi: M^{n} \rightarrow \Omega$ denotes the canonical projection and $\gamma: \mathbb{R} \rightarrow M^{n}$ is a normal 
geodesic parameterized by arc-length, then $\pi \circ \gamma$ maps $\mathbb{R}$ homeomorphically onto $\Omega$ in the first case, and it is a covering map of $\mathbb{R} \backslash\{0\}$ onto the subset $\Omega^{0}$ of internal points of $\Omega$ in the latter. Set $I=\gamma^{-1}(G(\mathcal{F}))$. Since $G(\mathcal{F})$ is a closed unbounded connected subset, using that $G(\mathcal{F})=G(\gamma(I))$ it follows easily that if $I \neq \mathbb{R}$ then $I=[a, \infty)$ for some $a \in \mathbb{R}$ in the first case and $I=(-\infty,-b] \cup[a, \infty)$ for some $a, b>0$ in the latter. Now observe that the type number of $f$ (i.e., the rank of its shape operator) is everywhere equal to 2 on $G(\mathcal{F})$. This is because the relative nullity subspace coincides with the nullity of the curvature tensor at a point where the type number is at least 2 , whence the subset where the type number is 2 is invariant under isometries. Let $\left(t_{0}-\varepsilon, t_{0}+\varepsilon\right) \subset I$ be such that $\Phi:\left(t_{0}-\varepsilon, t_{0}+\varepsilon\right) \times \Sigma_{p} \rightarrow \pi^{-1}\left(\left(t_{0}-\varepsilon, t_{0}+\varepsilon\right)\right)$ given by $\Phi(t, g(p))=g(\gamma(t)), p=\gamma\left(t_{0}\right)$, is a $G$-equivariant diffeomorphism. We call $\Gamma=\pi^{-1}\left(\left(t_{0}-\varepsilon, t_{0}+\varepsilon\right)\right)$ a tube around $\Sigma_{p}$. We have a well-defined vector field $\xi$ on $\Gamma$ given by $\xi(y)=g_{*}(\gamma(t)) \gamma^{\prime}(t)$ for $y=g(\gamma(t)), t \in\left(t_{0}-\varepsilon, t_{0}+\varepsilon\right)$, and $\xi(y)$ is orthogonal to $\Sigma_{\gamma(t)}$ at $y$.

Now let $\eta$ be a local unit normal vector field to $f$ on $\Gamma$ and $A_{\eta}^{f}$ the shape operator of $f$ with respect to $\eta$. Given a principal orbit $\Sigma_{q}=G(q) \subset \Gamma$ of $G$, the vector fields $\bar{\xi}=f_{*}\left(\left.\xi\right|_{\Sigma_{q}}\right)$ and $\bar{\eta}=\left.\eta\right|_{\Sigma_{q}}$ determine an orthonormal normal frame of the restriction $\left.f\right|_{\Sigma_{q}}: \Sigma_{q} \rightarrow \mathbb{R}^{n+1}$ of $f$ to $\Sigma_{q}$. Denote by $A_{\bar{\eta}}$ and $A_{\bar{\xi}}$ the corresponding shape operators. Notice that $A_{\bar{\xi}}=A_{\xi}^{i}$, where $i: \Sigma_{q} \rightarrow M^{n}$ is the inclusion of $\Sigma_{q}$ into $M^{n}$. Thus $A_{\bar{\xi}} \circ g_{*}=g_{*} \circ A_{\bar{\xi}}$ for any $g \in G$, hence the eigenvalues of $A_{\bar{\xi}}$ are constant. On the other hand, $A_{\bar{\eta}}=\Pi \circ A_{\eta}$, where $\Pi$ is the orthogonal projection of $T M^{n}$ onto $T \Sigma_{q}$. In particular, rank $A_{\bar{\eta}} \leq \operatorname{rank} A_{\eta}$, so we have rank $A_{\bar{\eta}} \leq 2$ on $\Sigma_{q}$. We have two cases to consider:

(i) $\operatorname{rank} A_{\bar{\eta}} \leq 1$ on each principal orbit contained in $\Gamma$;

(ii) $\operatorname{rank} A_{\bar{\eta}}=2$ on some principal orbit contained in $\Gamma$.

First we show that (i) can not occur. Assume otherwise. Then, it follows from Theorem 2 of [4] that the principal orbits in $\Gamma$ are either isometric to Euclidean spheres or isometrically covered by Riemannian products $\mathbb{R} \times S^{n-2}(a)$ (in what follows we suppose $a=1$ ). In the former case, for each principal orbit $\Sigma_{q} \subset \Gamma$ it follows from the Gauss equation of the restriction $\left.f\right|_{\Sigma_{q}}: \Sigma_{q} \rightarrow \mathbb{R}^{n+1}$ that $A_{\bar{\xi}}$ must be a multiple of the identity tensor, that is, the principal orbits in $\Gamma$ are umbilical in $M^{n}$. This is in contradiction with Lemma 2.8 of [9], taking into account that $n \geq 5$ and that $f$ has type number 2 on $\Gamma$.

Suppose now that the principal orbits are covered by $\mathbb{R} \times S^{n-2}$. In this case, for any fixed principal orbit $\Sigma_{q} \subset \Gamma$ there must exist an open subset $U_{0} \subset \Sigma_{q}$ where rank $A_{\bar{\eta}}=1$. In fact, otherwise $A_{\bar{\eta}}$ vanishes identically, hence the first normal spaces of $\left.f\right|_{\Sigma_{q}}$ (i.e., the subspaces of the normal spaces spanned by the image of the second fundamental form) have dimension one everywhere (notice that $A_{\bar{\xi}}$ can not vanish anywhere, otherwise it would be identically zero and $\left.f\right|_{\Sigma_{q}}$ would be totally geodesic, 
which is impossible). Then either $f\left(\Sigma_{q}\right)$ is contained in an affine hyperplane $\mathcal{H}$ of $\mathbb{R}^{n+1}$ or the first normal spaces of $\left.f\right|_{\Sigma_{q}}$ are nonparallel along an open subset of $\Sigma_{q}$. Both possibilities lead to contradictions: the latter forces $\Sigma_{q}$ to be flat (cf. [6], Theorem 1); in the former, since the shape operator of the isometric immersion $f: \Sigma_{q} \rightarrow \mathscr{H}$ is $A_{\bar{\xi}}$, which has constant eigenvalues, it follows that $f\left(\Sigma_{q}\right)$ is a round sphere, which is again impossible. We obtain that there exists an open subset $U \subset \Gamma$ where rank $A_{\bar{\eta}}=1$ and $U \cap \Sigma_{q}=U_{0}$. Since the images of $A_{\bar{\eta}}$ and $A_{\eta}$ are related by $\operatorname{Im}\left(A_{\bar{\eta}}\right)=\Pi\left(\operatorname{Im}\left(A_{\eta}\right)\right)$, and on $U$ the dimensions of $\operatorname{Im}\left(A_{\bar{\eta}}\right)$ and $\operatorname{Im}\left(A_{\eta}\right)$ are 1 and 2, respectively, we must have $\xi \in \operatorname{Im}\left(A_{\eta}\right)$ everywhere on $U$. Therefore, at any point $x \in U$ we have that $\operatorname{ker} A_{\eta}(x) \subset T_{x} \Sigma_{x}$, and hence $\operatorname{ker} A_{\eta}(x)=\operatorname{ker} A_{\bar{\eta}}(x)$. It follows that the leaves of the distribution on $U_{0}$ given by ker $A_{\bar{\eta}}$ are totally geodesic in $\Sigma_{q}$ and $\mathbb{R}^{n+1}$. In particular, they are flat hypersurfaces of $\Sigma_{q}$. This is in contradiction with the fact that $\Sigma_{q}$ is locally isometric to $\mathbb{R} \times S^{n-2}$. In fact, for any $x \in U_{0}$ let $W$ be an $(n-2)$-dimensional subspace of $T_{x}\left(\Sigma_{q}\right)$ where the sectional curvatures of $\Sigma_{q}$ are equal to 1 and let $F_{x}$ be the totally geodesic flat hypersurface through $x$. Then $S=W \cap T_{x}\left(F_{x}\right)$ has dimension at least 2 , since $n \geq 5$. At each bidimensional subspace of $S$, the sectional curvature of $\Sigma_{q}$ is 1, because $S \subset W$ and, on the other hand, such a curvature must be zero, for $S \subset T_{x}\left(F_{x}\right)$. Therefore (i) is not possible, and we are left with (ii).

If rank $A_{\bar{\eta}}=2$ along a principal orbit $\Sigma_{q} \subset \Gamma$, then rank $A_{\bar{\eta}}=2$ on a possibly smaller tube around $\Sigma_{q}$ contained in $\Gamma$, which we still denote by $\Gamma$. By Theorem 3 in [4], each principal orbit $\Sigma_{x}$ contained in $\Gamma$ is isometric to a Riemannian product $S^{2}(a) \times S^{n-3}(b)$ of spheres and $\left.f\right|_{\Sigma_{x}}: \Sigma_{x} \rightarrow \mathbb{R}^{n+1}$ splits as a product $\left.f\right|_{\Sigma_{x}}=$ $i_{1} \times i_{2}: S^{2}(a) \times S^{n-3}(b) \rightarrow \mathbb{R}^{3} \times \mathbb{R}^{n-2}=\mathbb{R}^{n+1}$, where $i_{1}: S^{2}(a) \rightarrow \mathbb{R}^{3}$ and $i_{2}: S^{n-3}(b) \rightarrow \mathbb{R}^{n-2}$ are umbilical inclusions. Moreover, $\{\bar{\eta}, \bar{\xi}\}$ is precisely the orthonormal normal frame of $\left.f\right|_{\Sigma_{x}}$ determined by the unit normal vector fields to the inclusions $i_{1}$ and $i_{2}$, respectively. In particular, $\bar{\xi}$ and $\bar{\eta}$ are parallel with respect to the normal connection of $\left.f\right|_{\Sigma_{x}}$. Hence, $A_{\bar{\eta}}$ coincides with the restriction of $A_{\eta}$ to $T \Sigma_{x}$, which in turn implies that $\xi$ is an eigenvector of $A_{\eta}$ along $\Gamma$. Now, since rank $A_{\eta}=\operatorname{rank} A_{\bar{\eta}}=2$ on $\Gamma$, it follows that $\xi \in \operatorname{ker} A_{\eta}$. Therefore, the segments of normal geodesics in $\Gamma$ are contained in the leaves of $\operatorname{ker} A_{\eta}$. Since these are assumed to be complete, we obtain that $f$ has type number 2 on the whole $M^{n}$ and that $A_{\eta}$ is everywhere of the form $A_{\eta}=\operatorname{diag}(\varphi, \varphi, 0, \ldots, 0)$, where $\varphi$ is nonzero and constant along each principal orbit and the $\varphi$-eigenspaces of $A_{\eta}\left(\right.$ or $\left.A_{\bar{\eta}}\right)$ coincide with $\operatorname{ker} A_{\bar{\xi}}=\operatorname{ker} A_{\xi}^{i}$. Now, let $X$ be a vector field such that $A_{\eta}(X)=\varphi X$. By the Codazzi equation

$$
\nabla_{X}\left(A_{\eta}(\xi)\right)-A_{\eta}\left(\nabla_{X} \xi\right)=\nabla_{\xi}\left(A_{\eta}(X)\right)-A_{\eta}\left(\nabla_{\xi} X\right)
$$

we get

$$
-A_{\eta}\left(\nabla_{X} \xi\right)=\xi(\varphi) X+\varphi \nabla_{\xi} X-A_{\eta}\left(\nabla_{\xi} X\right)=\xi(\varphi) X,
$$


where the last equality follows from $\nabla_{\xi} X \in\left(\operatorname{ker} A_{\eta}\right)^{\perp}=\operatorname{ker}\left(A_{\eta}-\varphi I\right)$, using that $\operatorname{ker} A_{\eta}$ is totally geodesic. Since $\nabla_{X} \xi=-A_{\xi}^{i}(X)=-A_{\bar{\xi}}(X)=0$, it follows that $\xi(\varphi)=0$. Therefore $\varphi$ is a constant, which we may suppose to be 1 . Standard arguments now show that $M^{n}$ splits as $M^{n}=S^{2} \times \mathbb{R}^{n-2}$ (cf. [13]). By the main lemma in [10], $f$ also splits as stated.

Proof of Theorem 1.1. Suppose that $f$ is not rigid. If $M^{n}$ is compact and $n \geq 3$, it follows from Theorem 2.1 that $B$, the set of totally geodesic points of $f$, disconnects $M^{n}$. In order to get the same conclusion in the non-compact case, we must show that there does not exist a complete leaf of relative nullity of $f$ of dimension $\ell=n-1$ or $\ell=n-2$. For $\ell=n-1$ this follows from our assumption on the flat part of $M^{n}$. Proposition 2.3 takes care of the case $\ell=n-2$.

Since $B$ disconnects $M^{n}$, it must contain a regular point $p$. Then the (principal) orbit $\Sigma$ through $p$ is contained in $B$, because $B$ is $G$-invariant by Proposition 2.2 (i). It follows from Lemma 3.14 of [5] that $f(\Sigma)$ is contained in a hyperplane $\mathscr{H}$ which is tangent to $f$ along $\Sigma$, for $\Sigma$ is connected. But $f(\Sigma)$ is an isoparametric hypersurface of a sphere by Proposition 2.2 (iii), and hence $f(\Sigma)$ must be a round hypersphere of $\mathscr{H}$. Proposition 2.2 (iv) now completes the proof.

Remark 2.4. In case $M^{n}$ is complete non-compact of dimension $n \geq 5$, the arguments in the beginning of the proof of Proposition 2.3 show, more precisely, that the conclusion of Theorem 1.1 fails only when every point of $G(\gamma(I))$ is flat, where $\gamma: \mathbb{R} \rightarrow M^{n}$ is a normal geodesic parameterized by arc-length and $I$ is either $[a, \infty)$ for some $a \in \mathbb{R}$ or $(-\infty,-b] \cup[a, \infty)$ for some $a, b>0$, according to the orbit space being homeomorphic to $\mathbb{R}$ or $[0, \infty)$, respectively. Notice that in the latter case $M^{n}$ is flat outside a compact subset. We also point out that, since $G$ is assumed to be compact, our assumption on the flat part of $M^{n}$ is equivalent to $M^{n}$ being unflat at infinity in the sense of [9].

Proof of Theorem 1.2. We already know from Theorem 1.1 that $f$ is either rigid or a hypersurface of revolution. Thus, by Proposition 2.2 (iv) it suffices to prove that any of the conditions in the statement implies that $f(\Sigma)$ is a round sphere for some principal orbit $\Sigma$ of $G$.

Assume first that $\Sigma$ is a positively curved principal orbit. By Proposition 2.2 (iii), $f$ immerses $\Sigma$ as a positively curved isoparametric hypersurface of some hypersphere of $\mathbb{R}^{n+1}$. It follows easily from the Cartan identities for isoparametric hypersurfaces of the sphere (cf. [4], Corollary 2) that $f(\Sigma)$ is a round sphere.

As for condition (ii), if $\Sigma$ is a totally geodesic principal orbit, then it is immersed by $f$ as an isoparametric hypersurface of a sphere $S^{n}$ whose first normal spaces in $\mathbb{R}^{n+1}$ are one-dimensional. This can only happen if it is umbilical in $S^{n}$, and hence again a round hypersphere of $S^{n}$. 
Now assume that (iii) holds. First notice that the position vector of $f$ can not be tangent to $f$ along $f(\Sigma)$ for every principal orbit $\Sigma$ of $G$, otherwise $f$ would be a cone over an isoparametric hypersurface of the sphere, in contradiction with the completeness of $M^{n}$. Now, if $\Sigma$ is a principal orbit along which the position vector is nowhere tangent to $f$, then the normal bundle of the restriction $\left.f\right|_{\Sigma}: \Sigma \rightarrow \mathbb{R}^{n+1}$ is spanned by the position vector and by $f_{*} \xi$, where $\xi$ is a unit normal vector field to the inclusion of $\Sigma$ into $M^{n}$. Since the shape operators of $\left.f\right|_{\Sigma}$ with respect to both vector fields are multiples of the identity tensor, it follows that $\left.f\right|_{\Sigma}$ is umbilical, and we obtain again that $f(\Sigma)$ is a round sphere.

Finally, under condition (iv) the conclusion is a consequence of the following result.

Proposition 2.5. Let $P^{n} \subset S^{n+1}, n \geq 4$, be an isoparametric hypersurface. If the universal covering of $P^{n}$ is (homeomorphic to) $S^{n}$, then $P^{n}$ is isometric to a Euclidean sphere.

Proof. Let $\lambda_{1}, \lambda_{2}, \ldots, \lambda_{g}$ be the distinct (and constant) principal curvatures of $P^{n}$. Let $m_{1}$ be the common multiplicity of the $\lambda_{k}$, when $k$ is odd, and let $m_{2}$ be the common multiplicity of the $\lambda_{k}$, when $k$ is even. Denote by $\beta_{0}, \beta_{1}, \beta_{2}, \ldots, \beta_{n}$ the $\mathbb{Z}_{2}$-Betti numbers of $P^{n}$. Then we have (cf. [11]):

(i) [F. Münzner] $g \in\{1,2,3,4,6\}$;

(ii) $2 n=g\left(m_{1}+m_{2}\right)$;

(iii) [E. Cartan] If $g=3$, then $m_{1}=m_{2} \in\{1,2,4,8\}$;

(iv) [U. Abresh] If $g=6$, then $m_{1}=m_{2} \in\{1,2\}$;

(v) [F. Münzner] $\sum_{i=0}^{n} \beta_{i}=2 g$.

Suppose first that $n$, the dimension of $P^{n}$, is odd. Then (ii) and (iv) imply that $g \in\{1,2,3\}$. Since $n \geq 4$, it follows from (iii) that $g \in\{1,2\}$. If $g=2$, then $P^{n}$ is a Riemannian product of spheres and thus it cannot be covered by a sphere. Hence we must have $g=1$ and this implies that $P^{n}$ is a Euclidean sphere.

Let now $n$ be even, say $n=2 q$. Then the Euler characteristics of $S^{2 q}$ and $P^{n}$ are related by $\chi\left(S^{2 q}\right)=m \chi\left(P^{n}\right)$, where $m$ is the number of sheets of the covering. Thus, either $m=1$ or $m=2$, since $\chi\left(S^{2 q}\right)=2$. Suppose $m=2$. Then $\chi\left(P^{n}\right)=\sum_{i=0}^{n}(-1)^{i} \beta_{i}=1$, which implies, using Poincare duality, that the Betti number $\beta_{q}$ is odd. On the other hand, we get from (v) that $\beta_{q}$ must be even. This contradiction tells us that $m=1$ and, again using (v), we obtain that $g=1$. Therefore $P^{n}$ is a Euclidean sphere.

Remark 2.6. Proposition 2.5 is no longer true for $n=3$, as shown by Cartan isoparametric hypersurfaces of $S^{4}$ with three distinct principal curvatures [3], which are diffeomorphic to $S^{3} / Q$, where $Q$ stands for the quaternion 8-group. 


\section{References}

[1] A. V. Alekseevsky, D. V. Alekseevsky, Riemannian $G$-manifold with one dimensional orbit space. Ann. Global Anal. Geom. 11 (1993), 197-211. Zbl 0810.53043 MR 1237453

[2] A.C.Asperti, F. Mercuri, M. H. Noronha, Cohomogeneity one manifolds and hypersurfaces of revolution. Boll. Un. Mat. Ital. B 11 (1997), 199-215. Zbl 0882.53006 MR 1456261

[3] E. Cartan, Familles de surfaces isoparamétriques dans les espaces à courbure constante. Ann. Mat. Pura Appl. 17 (1938), 177-191. Zbl 0020.06505

[4] H. P. Castro, M. H. Noronha, Homogeneous submanifolds of codimension two. Geom. Dedicata 78 (1999), 89-110. Zbl 0935.53023 MR 1722173

[5] M. Dajczer, D. Gromoll, Rigidity of complete Euclidean hypersurfaces. J. Differential Geom. 31 (1990), 401-416. Zbl 0667.53003 MR 1037409

[6] M. Dajczer, R. Tojeiro, Submanifolds with nonparallel first normal bundle. Canad. Math. Bull. 37 (1994), 330-337. Zbl 0812.53015 MR 1289768

[7] J.-H. Eschenburg, E. Heintze, On the classification of polar representations. Math. Z. 232 (1999), 391-398. Zbl 0943.22014 MR 1719714

[8] S. Kobayashi, Compact homogeneous hypersurfaces. Trans. Amer. Math. Soc. 88 (1958), 137-143. Zbl 0081.38102 MR 0096284

[9] F. Mercuri, J. A. P. Seixas, Hypersurfaces of cohomogeneity one and hypersurfaces of revolution. Differential Geom. Appl. 20 (2004), 255-239. Zbl 1046.53043 MR 2038557

[10] J. D. Moore, Isometric immersions of Riemannian products. J. Differential Geom. 5 (1971), 159-168. Zbl 0213.23804 MR 0307128

[11] R. Palais, C.-L. Terng, Critical Point Theory and Submanifold Geometry. Lecture Notes in Math. 1353, Springer-Verlag, Berlin 1988. Zbl 0658.49001 MR 0972503

[12] F. Podestà, A. Spiro, Cohomogeneity one manifolds and hypersurfaces of the Euclidean space. Ann. Global Anal. Geom. 13 (1995), 169-184. Zbl 0827.53007 MR 1336212

[13] P. Ryan, Homogeneity and some curvature conditions for hypersurfaces. Tôhoku Math. J. 21 (1969), 363-388. Zbl 0185.49904 MR 0253243

[14] R. Sacksteder, The rigidity of hypersurfaces. J. Math. Mech. 11 (1962), 929-939. Zbl 0108.34702 MR 0144286

Received April 13, 2005

Francesco Mercuri, Imecc-Unicamp, Campinas-SP, Brasil

E-mail: mercuri@ime.unicamp.br

Fabio Podestà, Math. Institute, Piazza Ghiberti, Firenze, Italy

E-mail: podesta@math.unifi.it

José A. P. Seixas, Mat-Ufal, Maceió-Al, Brasil

E-mail: adonai@mat.ufal.br

Ruy Tojeiro, Ufscar, São Carlos-SP, Brasil

E-mail: tojeiro@dm.ufscar.br 